\title{
2 Strategisch kommunizieren: In vier Phasen zum effektiven Kommunikationskonzept
}

Wiebke Lesch

- Kommunizieren heißt, wahrgenommen zu werden.

- Gerade bei kleinen Budgets sollte die Kommunikation strategisch geplant werden.

- Strategische Kommunikation besteht aus vier Phasen: Analyse, Strategie, Maßnahmen, Erfolgskontrolle.

- Die Analysephase hilft dabei, die Position der eigenen Organisation im Wettbewerbsumfeld zu bestimmen und die Bedürfnisse der Zielgruppen zu untersuchen.

- Die Strategie enthält Kommunikationsziele und -botschaften, die sich in einer kreativen Leitidee für die Kommunikation verdichten.

- Darauf aufbauend werden Maßnahmen und Kanäle passgenau für die Zielgruppen geplant und umgesetzt.

- Die Erfolgskontrolle gibt wichtige Aufschlüsse für die Planung zukünftiger Maßnahmen.

Im stark kompetitiven Umfeld von Wissenschaft und Forschung wird professionelle Kommunikation immer wichtiger. Sie unterstützt Wissenschaftseinrichtungen, Forschungsverbünde und Gesundheitseinrichtungen einerseits im Wettbewerb um knappe Fördermittel und Ressourcen, andererseits ist sie ein wichtiges Instrument für den Aufbau nationaler und internationaler Netzwerke. Insbesondere virtuelle Netzwerkorganisationen, die an vielen 
Standorten - national und/oder international - operieren, leben von Kommunikation, die es schafft, ein Gefühl der Präsenz und des Engagements seiner Mitglieder herzustellen.

Erfolgreiche Kommunikation unterstützt die Organisation bei der Erreichung ihrer Ziele und schafft es, einen vertrauensbildenden Dialog zwischen Wissenschaft, Gesellschaft und vor allem den internen und externen Bezugsgruppen der Organisation herzustellen.

Wissenschaftseinrichtungen, Forschungsverbünde und Gesundheitseinrichtungen verfügen im Vergleich $\mathrm{zu}$ anderen Branchen oft nur über kleine Budgets. Umso wichtiger ist eine gut geplante Strategie, wie welche Zielgruppe am besten erreicht wird, damit die knappen Mittel effektiv eingesetzt werden.

\section{Planen Sie Ihre Kommunikation!}

Sicher hat das jeder Kommunikationsverantwortliche schon einmal erlebt: Ein Kollege oder der Chef kommt auf Sie zu und fordert: „Machen Sie doch mal einen Flyer für die neue Studie. Wir müssen sie bei Wissenschaftlern bekannter machen!“ Im Alltagsdruck werden Maßnahmen oft schnell umgesetzt, bevor eine Strategie entwickelt und die Bedürfnisse der Zielgruppe genauer betrachtet wurden. Der Erfolg lässt dann oft zu wünschen übrig: Allein die Frage, wann, wo und wie der Flyer an die Wissenschaftler überreicht werden soll, zeigt schnell, dass dieses allzu beliebte Medium wahrscheinlich ungeeignet ist.

Wer langfristig zielführend und erfolgreich kommunizieren möchte, sollte strategisch vorgehen. Der klassische Prozess für die Planung einer Kommunikationsstrategie orientiert sich an vier Phasen:

1. Analyse

2. Strategie

3. Maßnahmen

4. Erfolgskontrolle

Dieser grundlegende Prozess ist für die Entwicklung einer Kommunikationsstrategie einer Forschungseinrichtung genauso anwendbar wie für eine Aufklärungskampagne. Man kann ihn auch mit dem Bau eines Hauses vergleichen: Zuerst errichtet man das Fundament (Analyse), dann die Mauern (Strategie) und schließlich das Dach (Maßnahmen). 


\subsection{Analyse: Sammeln Sie Informationen}

In der Analysephase werden die wesentlichen Daten gesammelt und verdichtet, auf denen die Kommunikationsstrategie aufbaut. Beschreiben Sie das vorliegende Problem bzw. die Aufgabe, welche gelöst werden soll und grenzen Sie es ein. Recherchieren Sie Wünsche, Meinungen und Verhaltensweisen der Stakeholder. Diese Informationen sind die Basis für das weitere Vorgehen.

Soll eine Kommunikationsstrategie für ein Forschungszentrum entwickelt werden, dann könnten folgende Kernfragen am Anfang der Analysephase stehen:

- Wie wird die eigene Organisation und ihr Leistungsangebot von den relevanten Stakeholdern gesehen?

- Welche (kommunikativen) Stärken und Schwächen weist die Organisation auf?

- Wie grenzt sie sich in ihrem Image und ihrem Leistungsspektrum von ihrem Wettbewerbsumfeld ab?

- Wo liegt das Potenzial für ein (kommunikatives) Alleinstellungsmerkmal?

Weiterhin werden die Zielgruppen und ihre Bedürfnisse genau identifiziert. Versuchen Sie, so viel wie möglich über die Zielgruppen herauszufinden (s.a. Kap. 1.2.3 „Zielgruppen“). Mögliche Wege sind Interviews mit bzw. Befragungen von Stakeholdern und/oder Kunden. Je nach Aufgabenstellung und Datenlage eignen sich beispielsweise quantitative Befragungen, qualitative Interviews oder Fokusgruppen mit Nutzern. Experteninterviews können hilfreich sein, um einen schnellen Überblick zu einem Thema zu bekommen und um die Historie, den Kontext und Veränderungen in einem Feld zu erfassen.

Ergänzend können Hintergrundinformationen mithilfe von Sekundärdaten (Studien, Literatur, Medienanalysen, Datenbanken und Archive, Gesetzestexte etc.) eingeholt werden. Weiterhin existieren viele hilfreiche betriebswirtschaftliche Analyseinstrumente, die dabei helfen können, eine Problemstellung präzise zu beschreiben. Eine in der Praxis sehr beliebte Analysemethode ist die SWOT-Analyse. Die Abkürzung SWOT steht für: Strengths (Stärken), Weaknesses (Schwächen), Opportunities (Chancen) und Threats (Risiken). Sie stellt eine Matrix zur Erfassung der organisationseigenen Stärken und Schwächen sowie der umfeldbedingten Chancen und Risiken dar (Bruhn 2009).

Vergangene Kommunikationsmaßnahmen der eigenen Organisation sollten ebenfalls analysiert und deren Erfolg bewertet werden. 


\subsection{Strategie: Bestimmen Sie, was Sie wie tun wollen}

"The essence of strategy is choosing to perform activities differently than rivals do." (Michael E. Porter)

Die Kommunikationsstrategie beginnt mit der Definition strategischer Ziele, die sich von den Unternehmens- und/oder Marketingzielen ableiten (Dörrbecker u. Fissenewert-Gossmann 2003). Strategische Ziele sind mit den organisationsbzw. netzwerkspezifischen Zielen, der Vision und der Organisationskultur zu synchronisieren und können nicht losgelöst davon betrachtet werden.

\subsubsection{Kommunikationsziele}

Wenn Sie nicht wissen, was Sie erreichen wollen, wird es Ihnen schwer fallen festzulegen, wie sie es tun sollen. Definieren Sie deshalb Ihr Kommunikationsziel, beispielsweise:

- Bekanntheit bei definierten Bezugsgruppen erhöhen

- Awareness und Involvement von Zielgruppen erreichen

- Image bei definierten Zielgruppen verbessern

- Verhaltensänderung und/oder Wissensvermittlung zu bestimmten Themen erreichen

- Beziehungspflege zu Medienvertretern, um eine positive Berichterstattung zu unterstützen

Die Ziele sind je nach Problemstellung individuell zu entwickeln. Steht eine Forschungsinitiative noch ganz am Anfang, wird der Fokus darauf liegen, Bekannheit in den wichtigsten Stakeholdergruppen zu erzeugen und um Akzeptanz zu werben. Ist die Organisation schon gut etabliert, kann es darum gehen, neue Zielgruppen zu erschließen oder das Markenimage zu verändern.

Ziele sollten immer möglichst messbar definiert und differenziert werden (Dörrbecker u. Fissenewert-Gossmann 2003; Bruhn 2003).

\subsubsection{Positionierung}

Auf der Grundlage der zuvor erfassten Daten der Situationsanalyse lässt sich im nächsten Schritt eine kommunikative Positionierung im Organisationsumfeld vornehmen. Dazu müssen drei einfache Fragen kurz und präzise beantwortet werden (Neumeier 2005):

1. Who are you? (Wer bist du?)

2. What do you do? (Was tust du?)

3. Why does it matter? (Warum ist das relevant?) 
Die Beantwortung dieser einfachen Fragen ist oft schwerer als man auf den ersten Blick denkt. Je kompetitiver ein Markt ist, umso mehr Einfluss hat die Positionierung auf den Erfolg oder Misserfolg einer Marke. In der Medizin ist diese Denkweise noch ungewohnt, kann aber auch dort gewinnbringend eingesetzt werden. Die knappe und klare Beantwortung der Positionierungsfrage ist für die interne und externe Kommunikation sehr wichtig. Als Hilfestellung kann der von Neumeier stammende Satz in den eckigen Klammern komplettiert werden:

$$
\text { „Our [offerenig] is the only [category] that [benefit].“ (Neumeier 2014) }
$$

Ein Beispiel könnte sein: Unsere Biobank für Herz-, Kreislauferkrankungen (BIOKARD) ist die einzige Biobank, die bundesweit Wissenschaftlern Biomaterialproben für die akademische Herz-, Kreislaufforschung zur Verfügung stellt.

\subsubsection{Zielgruppen}

Die Zielgruppen einer Organisation (synonym auch Stakeholder, Teilöffentlichkeiten oder Dialoggruppen genannt) sind die Gesamtheit aller effektiven oder potenziellen Personen, die mit einer bestimmten Maßnahme angesprochen werden sollen (Gabler Wirtschaftslexikon 2014).

$\mathrm{Zu}$ den Zielgruppen im Gesundheits- und Forschungsbereich gehören die in Tabelle 1 dargestellten.

\section{Tab. 1 Zielgruppen im Gesundheits- und Forschungsbereich}

\begin{tabular}{|c|c|c|c|}
\hline Fachzielgruppen & $\begin{array}{l}\text { Nichtfachliche } \\
\text { Zielgruppen }\end{array}$ & $\begin{array}{l}\text { Organisationsspezifische } \\
\text { Zielgruppen }\end{array}$ & Weitere \\
\hline $\begin{array}{l}\text { Wissenschaftler an } \\
\text { Universitäten und For- } \\
\text { schungseinrichtungen } \\
\text { Niedergelassene Ärzte } \\
\text { Kliniker (nach Fach- } \\
\text { gebiet) } \\
\text { - Epidemiologen } \\
\text { - Fachgesellschaften } \\
\text { - Pharma- und Medizin- } \\
\text { technikunternehmen }\end{array}$ & $\begin{array}{l}\text { Patienten } \\
\text { Eltern } \\
\text { (Pflegende) } \\
\text { Angehörige } \\
\text { Öffentlichkeit mit } \\
\text { speziellen Fragen } \\
\text { zu Gesundsheitsri- } \\
\text { siken etc. } \\
\text { Patientenverbände }\end{array}$ & $\begin{array}{l}\text { Mitarbeiter } \\
\text { Gremien } \\
\text { Mitglieder (wie z.B. Ärzte, } \\
\text { Study nurses, Forschungs- } \\
\text { gruppen) } \\
\text { Verbundpartner } \\
\text { Vorstände } \\
\text { Beiräte } \\
\text { Forschungsförderer/Geld- } \\
\text { geber }\end{array}$ & $\begin{array}{l}\text { Politik } \\
\text { - Medien }\end{array}$ \\
\hline
\end{tabular}

Eine Stakeholder-Analyse ist für den Einstieg sinnvoll, um systematisch zu erfassen, welche Zielgruppen für den Erfolg der Organisation wichtig sind und um Prioritäten zu setzen. Aufgrund limitierter Ressourcen können häufig nicht alle Gruppen gleichermaßen angesprochen werden. 


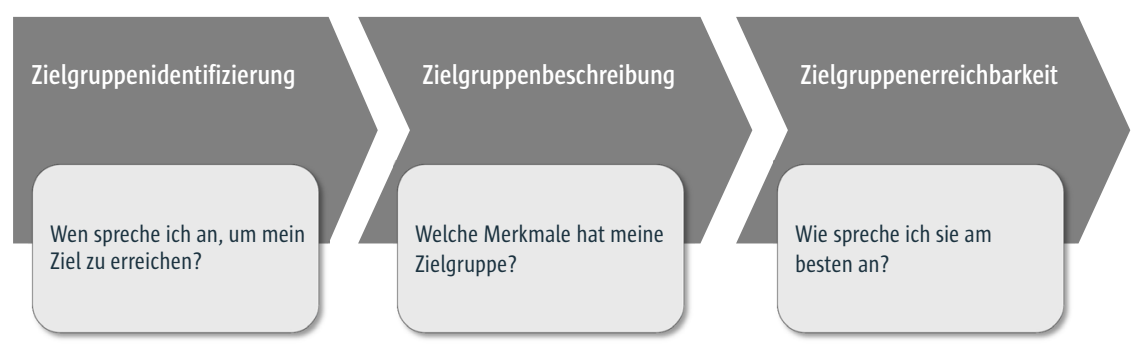

Abb. 1 Zielgruppenplanung

Im nächsten Schritt sollten die Zielgruppen möglichst genau differenziert und beschrieben werden (s. Abb. 1). Sozio-demografische Merkmale sind nur eine Grundlage für eine Zielgruppenbeschreibung, aber sie sind längst nicht ausreichend und können sogar irreführend sein. Das wird an dem folgenden, sehr plakativen Beispiel deutlich: Der britische Thronfolger Prinz Charles und der britische Rockmusiker Ozzy Osbourne sind zwei Männer, 1948 in Großbritannien geboren, verheiratet, haben erwachsene Kinder, sind beruflich erfolgreich und vermögend. Sie sind sozio-demografische Zwillinge und könnten aber in ihren Einstellungen und Motivationen verschiedener kaum sein.

Um aus der Sicht der Zielgruppe zu agieren und passgenaue Kommunikationsangebote und Services bereitzustellen, ist es unabdingbar, Einstellungen und Motivationen der Zielgruppe zu kennen:

- Wie nimmt meine Zielgruppe die Organisation bzw. das Angebot wahr?

- Was sind Stärken, was sind Schwächen?

- Welche Medien und Kanäle werden von der Zielgruppe bevorzugt genutzt?

Um Einstellungen und Motivationen der Zielgruppe herauszubekommen, muss man sich meistens aus den eigenen vier Wänden herausbewegen und die Perspektive der Zielgruppe einnehmen. Wenn Sie mit Ärzten als Forschungspartner arbeiten, dann nehmen Sie sich die Zeit und schauen Sie sich den Forschungsalltag im Krankenhaus oder Labor an. Nehmen Sie an einem Patientenaufklärungsgespräch teil und sprechen Sie mit Patienten, um deren Probleme zu verstehen. Schauen Sie einer Study Nurse über die Schulter, um die Herausforderungen ihres Alltags zu begreifen. Versuchen Sie, sich in die jeweilige Person hineinzuversetzen und lernen Sie, was ihre Zielgruppe motiviert oder warum sie sich auf eine ganz bestimmte Art und Weise verhält.

Nutzerinterviews, Fokusgruppen und ethnografische Forschungsmethoden sind hilfreiche Instrumente, um eine qualitative und menschzentrierte Zielgruppenrecherche zu betreiben. Quantitative Daten wie das Mediennutzungsverhalten größerer demografischer Gruppen sind je nach Aufgabenstel- 
lung ebenfalls nützlich und können über Recherchen in der Fachliteratur, aktuelle Studien und in fachbezogenen Datenbanken gefunden werden.

\section{Checkliste: Kennen Sie Ihre Zielgruppen?}

1. Haben Sie Ihre wichtigste(n) Zielgruppe(n) festgelegt?

Behalten Sie immer Ihre Zielgruppe(n) und deren Bedürfnisse im Auge. Segmentieren Sie sie auf sinnvolle Art und Weise: Welche haben die höchste Priorität? Welche kann man am einfachsten erreichen? Welche sind groß genug, um eine Maßnahme zu rechtfertigen?

2. Haben Sie Forschung zu Ihrer Zielgruppe betrieben? Kennen Sie Ihre Zielgruppe! Treffen Sie keine Entscheidungen auf Basis von Annahmen oder Vermutungen. Schauen Sie sich vorhandene Daten an oder sammeln Sie neue, eigene Daten. Während dieser Phase ist es sinnvoll, den Wissensstand, die Einstellungen sowie die Wünsche und Bedürfnisse der Zielgruppe genauer zu erforschen und zu verstehen. Je mehr Sie über Ihr Zielgruppensegment wissen, umso besser können Sie es mit Ihren Botschaften oder Aktivitäten erreichen.

3. Haben Sie Ihre Ziele in Bezug auf die gewünschte Verhaltensänderung und die Kernbotschaften identifiziert?

Was wollen Sie bei Ihrer Zielgruppe erreichen? Was soll sie tun? Definieren Sie klare Ziele auf Basis verhaltenstheoretischer und kommunikationswissenschaftlicher Erkenntnisse.

4. Haben Sie festgelegt, wie Ihr Material gestaltet und verteilt werden soll, damit es Ihre Zielgruppe erreicht?

Wenn die Maßnahme oder das gestaltete Material die Zielgruppe nicht erreicht, das gewählte Format von der Zielgruppe nicht genutzt oder verstanden wird, dann haben Sie Ihr Ziel verfehlt. Wählen Sie den besten Kanal (Print, Video, auditives Medium etc.), um Ihre Zielgruppe zu erreichen. Wenn Sie online Material bereitstellen, beachten Sie gängige Usabilityprinzipien. Identifizieren Sie geeignete Distributionskanäle wie Social Media, gemeinnützige Organisationen, Webseiten oder Events, die Ihre Zielgruppe nutzt.

5. Haben Sie Zeit und Ressourcen eingeplant, um Ihr Material in der Zielgruppe zu testen und auf Basis dieses Feedbacks anzupassen?

Dieser Schritt kann mehrfach wiederholt werden. Er ist äußerst wertvoll, um die Wirksamkeit der Maßnahme oder des Materials zu testen.

(Quellen: CDC Clear Communication Index, Appendix A: Developing Effective Communication Products; CDCynergy health communication model)

\subsubsection{Botschaften}

Die Kommunikationsbotschaften sind die Inhalte der Kommunikation, also das, was in den Köpfen der Zielgruppen ankommen soll. Je nach Zielgrup- 
pe unterscheiden sie sich in ihrer Informationstiefe und Tonalität. Die Botschaften sind an die Sprache der Empfänger angepasst. Sie sind der „Rohstoff “ für die spätere Gestaltung der Kommunikationsmaterialien, also noch keine ausgefeilten Texte. Bei der Formulierung einer Botschaft gilt das KISSPrinzip: keep it short and simple. Die einzelnen Kommunikationsbotschaften sollten $\mathrm{zu}$ einer Leitidee verdichtet werden, die die zentrale Botschaft ausdrückt. An ihr orientieren sich alle Kommunikationsmaßnahmen.

\subsubsection{Briefing}

Alle vorher gesammelten Informationen fließen schließlich in einem Briefing zusammen. Ein Briefing ist ein Papier, das alle Fakten, Hintergründe, Meinungen und Agendas zusammenfasst, die im Zusammenhang mit der Aufgabe von Bedeutung sein können. Dazu gehören auch weiche Fakten wie die persönliche Meinung des Auftraggebers oder Vorgesetzten.

Ein vollständiges Briefing enthält:

- eine Beschreibung der Aufgabe

- Ziele

- Zielgruppen

- Positionierung

- Kommunikationsbotschaften

- Tonalität

- Leistungsumfang/Anforderungen an die Maßnahmen

- Vorgaben zum Corporate Design

- Terminplanung

- Budgetrahmen

Für die Zusammenarbeit mit einer externen Agentur ist ein schriftliches Briefing unabdingbar. Nur mit einem guten Briefing werden Sie ein Ergebnis bekommen, das ihre Aufgabenstellung bestmöglich löst.

Manchmal führt die Situationsanalyse zu einem Re-Briefing, d.h. zu einer veränderten Aufgabenstellung. Das kann dann passieren, wenn die Aufgabenstellung nicht präzise und differenziert genug formuliert oder nicht realistisch war. Der Kommunikationsverantwortliche hat hier eine Beraterfunktion, die er durch die intensive Auseinandersetzung mit der Ist-Situation und das Sammeln von Fakten untermauert.

\subsection{Maßnahmen und Kanäle: So setzen Sie Kommunikation gelungen um}

Nun kann es an die Planung der Maßnahmen und Kanäle gehen. Sie werden im Kommunikationsplan festgelegt. Der Kommunikationsplan ist ein 
umfassender strategischer Handlungsplan, der angestrebte Ziele erreichen möchte, für ihre Umsetzung geeignete Strategien wählt und darauf aufbauend adäquate Kommunikationsinstrumente festlegt (Becker 2001). Maßgeblich für die Auswahl der Maßnahmen ist, welche internen und externen Kommunikations- und Medienaktivitäten die Ziele der Organisation oder das Kampagnenziel am besten unterstützen und die Zielgruppen am effizientesten erreichen. Sie variieren je nach Aufgabenstellung und können sein:

- Digitale Kommunikation (Webseiten, Blogs, Microsites etc.)

- Social Media (Facebook, Twitter, LinkedIn, Xing, Instagram etc.)

- Print und- Mediawerbung (Anzeigen, Flyer, Broschüren, Spots etc.)

- Direct Marketing (Mailings etc.)

- Public Relations (PR)

- Event Marketing (Fachkongresse und Messen)

- Lobbying

- Virales Marketing

- Guerilla Marketing

Welche Maßnahmen und Kanäle gewählt werden, hängt entscheidend von den Informationsbedürfnissen und Präferenzen der anvisierten Zielgruppe(n) $\mathrm{ab}$.

\subsubsection{Kreative Leitidee}

Sind die geeigneten Maßnahmen und Kanäle ausgewählt, braucht es im nächsten Schritt eine kreative Leitidee, die die Botschaften visuell und textlich verdichtet. Das kann in Form eines visuellen (Key Visual) oder textlichen Elements (Slogan) sein oder als Kombination aus beidem. Wichtig ist, dass dieses Element einen hohen Wiedererkennungswert hat und zum Markenkern passt.

Eine Maßnahme sollte immer zur Organisations- bzw. Markenidentität passen. Wird der Markenkern als sachlich und seriös definiert, dann passt eine laute Tonalität oder ein provokatives Key Visual schlecht dazu. Hieran wird deutlich, wie wichtig der vorangegangene strategische Prozess ist. Wenn der Markenkern nicht definiert ist, wird die Auswahl einer kreativen Leitidee schnell zur „geschmäcklerischen“ Diskussion. Weiterhin sollte immer geprüft werden, ob sich die Leitidee auf verschiedene Medien im Sinne einer integrierten Kommunikation übertragen lässt.

Am Ende werden alle geplanten Maßnahmen in einer zeitlichen Dramaturgie, einem Maßnahmenplan, zusammengefasst, aus dem hervorgeht, wann welche Zielgruppen wo und mit welchen Maßnahmen erreicht werden sollen. Man nennt das auch: „Management der Touchpoints“. Der Maßnahmenplan stellt sicher, dass eine Maßnahme oder Intervention konsistent von der Zielgruppe wahrgenommen wird. Entscheidend ist, dass alles gut koordiniert und aufeinander abgestimmt sind. 
Anhand des Kommunikationsplans kann auch das Budget errechnet werden, welches für die Interventionen aufgewendet werden muss. Bei der Auswahl der Maßnahmen sollte nicht vergessen werden, die eigenen personellen, finanziellen und sachlichen Ressourcen richtig einzuschätzen, um Fehlentscheidungen zu vermeiden.

\section{Praktische Tipps für effektive Kommunikationsmaterialien}

Hauptbotschaft und Handlungsaufforderung („Call to action“)

1. Enthält das Kommunikationsmaterial eine Hauptbotschaft?

Stellen Sie sicher, dass Ihr Kommunikationsmaterial eine Hauptbotschaft enthält. Die Hauptbotschaft ist das, woran sich Ihre Zielgruppe erinnern soll. Sie kann auch mit dem „call to action“ (das, was die Empfänger tun sollen, nachdem sie die Hauptbotschaft wahrgenommen und verstanden haben) zusammengefasst werden.

2. Steht die Hauptbotschaft ganz oben oder am Anfang des Kommunikationsmaterials?

Menschen suchen nach der wichtigsten Information ganz oben oder am Anfang des Kommunikationsmaterials. Stellen Sie deshalb die Hauptbotschaft an den Anfang oder bringen Sie sie auf dem Titel unter, damit sie leichter gefunden wird. Auf einer Webseite gehört die Hauptbotschaft z.B. in den Kopf oder in den ersten Abschnitt der Seite, die ohne Scrollen erreichbar ist. Bei einem Flyer würde man sie auf dem Titel unterbringen.

3. Wird die Hauptbotschaft mit visuellen Elementen unterstützt? Visuelle Elemente (Farben, Bilder, Formen, Linien, Schriften etc.) lenken die Aufmerksamkeit des Betrachters auf bestimmte Bereiche. Nutzen Sie visuelle Elemente, um den Betrachter auf die Hauptbotschaft aufmerksam zu machen.

4. Enthält das Material wenigstens ein Bildelement, welches die Hauptbotschaft unterstützt?

Stellen Sie sicher, dass Text- und Bildelemente dieselbe Botschaft transportieren und sich gegenseitig verstärken. Der Betrachter erwartet, dass Texte und Bilder, die unmittelbar nebeneinander stehen, ähnliche Informationen enthalten. Haben sie keinen Bezug oder sind sogar widersprüchlich, dann stiftet das Verwirrung oder lenkt ab. Fotos, Grafiken und Infografiken sind gut geeignet, Informationen leichter zu vermitteln.

5. Enthält das Material eine oder mehrere Handlungsaufforderungen für die Zielgruppe?

Machen Sie Ihrer Zielgruppe deutlich, was sie mit denen von ihnen vermittelten Informationen tun sollen. Möchten Sie, dass sich jemand bei Ihnen meldet? Oder möchten Sie weitere Informationen? Möchten Sie eine Verhaltensänderung bewirken? Selbst, wenn Sie nur über etwas informieren, sollten Sie darüber nachdenken, warum die Zielgruppe diese Informationen gebrauchen könnte, und diese Erkenntnisse nutzen, um eine Handlungsaufforderung daran zu koppeln. 


\section{Sprache}

- Sind Hauptbotschaft und "Call to action" in aktiver Sprache formuliert? Vermeiden Sie passive, negative oder abstrakte Formulierungen. Ersetzen Sie Substantive durch Verben, passive durch aktive Formulierungen („wurde beschlossen“ durch „beschloss") und vermeiden Sie Fremdwörter.

- Sprechen Sie die Sprache ihrer Zielgruppe?

Schreiben Sie in der Sprache, die Ihre Zielgruppe im Alltag spricht. Dann verarbeiten die Empfänger die Informationen einfacher und schneller. UserTests zeigen, ob man die richtige Tonalität getroffen hat. Muss ein Fremdwort verwendet werden, dann erklären Sie es. Bevorzugen Sie einfache Verben und Substantive gegenüber Fachbegriffen.

\section{Informationsdesign}

- Verwenden Sie Listen und/oder Aufzählungen?

Verwenden Sie Listen, um Fließtexte leichter zugänglich und schneller erfassbar zu gestalten. Listen mit mehr als sieben Punkten sollten unterteilt werden.

- Ist der Text in Abschnitte mit Zwischenüberschriften aufgeteilt?

Unterteilen Sie Texte in kleine Abschnitte, die mit Zwischenüberschriften unterteilt werden. Ein Abschnitt sollte eine Sinneinheit darstellen und nur so viele Informationen enthalten, wie Menschen in ihrem Kurzzeitgedächtnis behalten können. Testen Sie es! Zwischenüberschriften müssen in Kurzform genau die Informationen enthalten, die im folgenden Abschnitt enthalten sind. Alles andere verwirrt Ihre Leser.

- Steht die wichtigste Information im ersten Abschnitt?

Strukturieren Sie Informationen so, dass Sie Ihre Zielgruppe nicht überfordern. Sie müssen nicht alle vorhandenen Informationen in einem Kommunikationsmaterial unterbringen! Konzentrieren Sie sich stattdessen auf die wichtigsten Informationen, die die Hauptbotschaft unterstützen. Sie können an geeigneter Stelle auf weiterführende Informationen verweisen.

\section{Aktueller Wissensstand}

- Erklären Sie den aktuellen Wissensstand zum Thema und gehen Sie auch auf Unsicherheiten ein?

Wissenschaftliche Erkenntnisse entwickeln sich stets weiter. Erklären Sie, wo Unsicherheiten und Limitationen bei Erkenntnissen, Daten oder Empfehlungen bestehen.

(Quelle: CDC Clear Communication Index, Appendix A: Developing Effective Communication Products)

\subsection{Erfolgskontrolle: War Ihre Strategie erfolgreich?}

Sind alle Maßnahmen umgesetzt, dann ist es an der Zeit, den Erfolg der Intervention zu überprüfen. Der Erfolg strategisch geplanter Kommunika- 
tion lässt sich anhand bestimmter Kennzahlen wie Umfragen, Responsedaten oder Balanced Scorecards messen.

Abhängig von der Aufgabenstellung und Zielgruppe lassen sich Bekanntheit, Image und der Grad der Implementation einer Botschaft in der Zielgruppe über Befragungen messen. Der Aufwand hierfür ist im Verhältnis zu den kleinen Budgets in Forschungsinstitutionen aber relativ hoch und ihre Sinnhaftigkeit muss vor dem Hintergrund der Aufgabenstellung bewertet werden. Der Erfolg von Direct Mailings lässt sich anhand von Responseraten bestimmen, eine Online-Werbekampagne anhand von Klickraten und der Migration der Besucher, eine Facebookkampagne anhand der Likes und des ROI usw. Jedes Medium hat seine eigenen Kennzahlen, was die Evaluation einer Multi-Channel-Marketingaktion relativ komplex macht. Über die Kennzahlen der Evaluation sollten Sie sich schon bei der Planung der Maßnahmen Gedanken machen und diese auch budgetieren.

\subsection{Zusammenfassung}

Kommunikation braucht unabhängig von der Größe des Kommunikationsbudgets eine Strategie. Nur wer weiß, welche Zielgruppe er wann und mit welchen Maßnahmen erreichen möchte, kann zielgerichtet und erfolgreich

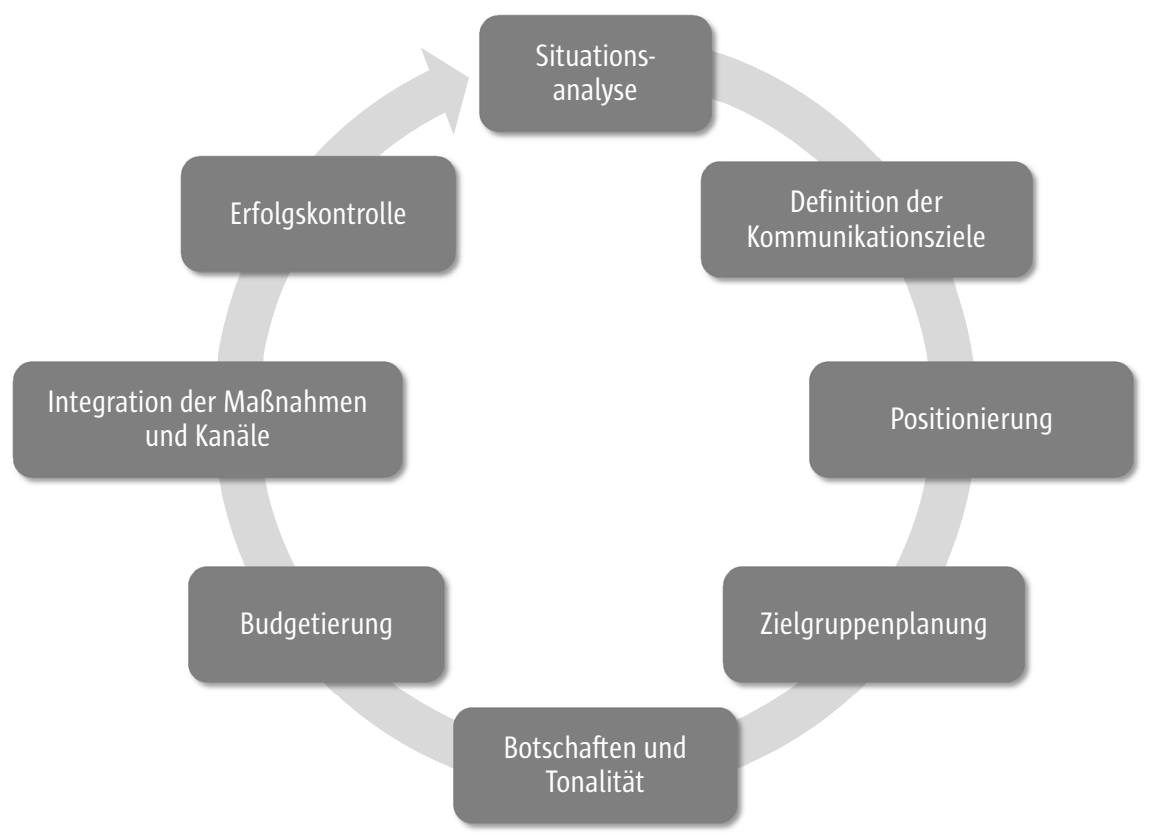

Abb. 2 Die Kernelemente eines Kommunikationskonzeptes im Überblick 
kommunizieren. Alle Maßnahmen sollten eng koordiniert und aufeinander abgestimmt werden.

Am Anfang einer Kommunikationsstrategie wird die Aufgabenstellung oder das Problem beschrieben, welches mittels kommunikativer Maßnahmen gelöst werden soll. In dieser anfänglichen Analysephase werden Daten gesammelt, entweder aus Sekundärquellen oder durch eigene Forschung. Fassen Sie aus den Analyseergebnissen die Faktoren zusammen, die das Projekt beeinflussen könnten, und listen Sie die Ursachen der Probleme auf, die Sie mit der Kommunikationsmaßnahme lösen wollen. Entwickeln Sie daraus die Kommunikationsziele für jedes Problem.

Unabhängig davon, ob Kommunikation als Hauptintervention (z.B. eine Aufklärungskampagne) oder unterstützend für ein Produkt oder einen Service eingesetzt wird, müssen zunächst die Zielgruppen bestimmt und nach Priorität segmentiert werden. Für jedes Segment sollten Kommunikationsziele festgelegt werden. Im Briefing wird schließlich das Konzept zusammengefasst: Dazu gehören die Kommunikationsbotschaften, die Tonalität, die Maßnahmen und Kanäle, die Kommunikationsmaterialien sowie ein Zeit-, Ressourcen- und Budgetplan. Entwickeln Sie idealerweise die Konzepte, Botschaften, Maßnahmen und Materialien gemeinsam mit Ihrer Zielgruppe (s. Abb. 2). Falls das nicht möglich ist, dann testen Sie sie in der Zielgruppe und arbeiten das Feedback ein. Die Evaluation misst schließlich den Erfolg der Kommunikation.

\section{Quellenangaben}

Becker (2001): Marketing-Konzeption: Grundlagen des strategischen Marketing-Managements, 7. überarbeitete und ergänzte Auflage, München, Vahlen

Bonk/Griggs/Tynes (2007): The Jossey-Bass guide to strategic communications for nonprofits. Jossey-Bass; $1^{\text {st }}$ edition

Bruhn (2003): Kommunikationspolitik, 2. Auflage, München, Vahlen

Bruhn (2009): Übungsbuch zur Kommunikationspolitik: Basiswissen, Aufgaben und Lösungen, 1. Auflage, Vahlen Centers for Disease Control and Prevention (2016): CDCynergy health communication model. Stand: 27.01.2016. http:// www.orau.gov/cdcynergy/web/BA/Content/phase3/phase3mainframeset.htm (abgerufen am 27.01.2016)

Centers for Disease Control and Prevention (2016): The CDC Clear Communication Index. Stand: 10.11.2015. http://www.cdc.gov/ccindex/ (abgerufen am 27.01.2016)

Dörrbecker/Fissenewert-Gossmann (2003): Wie Profis PR-Konzeptionen entwickeln, 4. Auflage, Frankfurt am Main, Frankfurter Allgemeine Buch

Gabler Wirtschaftslexikon (2014), Verlag: Springer Gabler; Auflage: 18., aktualisierte Auflage 2014

Neumeier (2005): The Brand Gap: How to Bridge the Distance Between Business Strategy and Design, New Riders; Revised and Expanded Edition edition

Neumeier(2014):StealThisIdea:BrandPositioning,Stand:07.01.2014.http://www.slideshare.net/liquidagency/ steal-this-idea-brand-positioning (abgerufen am 16.02.2016) 